\title{
Intramedullary nailing of tibial non-unions using the suprapatellar approach: a case series
}

\author{
Botma $\mathrm{N}^{1} \oplus$, Graham $\mathrm{S}^{2}{ }^{\oplus}$, Held $\mathrm{M}^{3} \mathbb{D}^{\circ}$, Laubscher $\mathrm{M}^{4}$ \\ $1 \mathrm{MBChB}($ Stell); Orthopaedic registrar \\ 2 MBChB, MRCS, MSc(Res), FRCS(Tr\&Ortho); Orthopaedic surgeon \\ 3 MD, PhD, FC Orth(SA); Orthopaedic surgeon \\ 4 MBChB(UFS), Dip PEC, FC Orth(SA), MMed Ortho(UCT); Orthopaedic surgeon
}

Orthopaedic Research Unit, Department of Orthopaedic Surgery, Groote Schuur Hospital, University of Cape Town, South Africa

Corresponding author: Dr Nico Botma, Orthopaedic Research Unit, Department of Orthopaedic Surgery, H49 OMB, Groote Schuur Hospital, University of Cape Town, Cape Town, 7925, South Africa; tel: (021) 404 9111; email: nico.botma@gmail.com

\begin{abstract}
Background: A number of treatment options are available for diaphyseal non-unions of the tibia, including intramedullary (IM) nailing. An infrapatellar entry point with the knee in deep flexion can make this procedure challenging, especially with associated deformity or an obliterated canal. The suprapatellar approach allows nail insertion with the knee extended, which facilitates correction of malalignment in the sagittal and coronal planes. The aim of our study was to review the outcome of diaphyseal tibial non-unions, treated with an intramedullary nail, using the suprapatellar approach.

Method: We retrospectively reviewed consecutive cases with non-union of the tibial shaft, treated with a suprapatellar entry nail between May 2016 and January 2018. Patients who were previously managed with a nail or who had active sepsis were excluded. The rate and time to union, as well as complications were assessed.

Results: Thirteen cases were included and followed up until union at a mean of 5.8 months. All were performed percutaneously, without opening of the non-union site. Two patients developed complications, although bony union was still achieved.

Conclusion: A suprapatellar entry tibial nail is an acceptable treatment option for tibial non-unions not previously treated with a nail.
\end{abstract}

Level of evidence: Level 4

Keywords: tibia, non-union, suprapatellar, intramedullary, nail

Citation: Botma N, Graham S, Held M, Laubscher M. Intramedullary nailing of tibial non-unions using the suprapatellar approach: a case series. SA Orthop J 2020;19(1):40-45. http://dx.doi.org/10.17159/2309-8309/2020/v19n1a6

Editor: Prof. N Ferreira, Stellenbosch University, South Africa

Received: March 2019

Accepted: September 2019

Published: March 2020

Copyright: () 2020 Botma N, et al. This is an open-access article distributed under the terms of the Creative Commons Attribution Licence, which permits unrestricted use, distribution and reproduction in any medium, provided the original author and source are credited.

Funding: There are no funding sources to declare.

Conflict of interest: All authors confirm they have no conflicts of interest to declare with regard to this article. 


\section{Introduction}

The reported incidence of tibial shaft non-union ranges from 4 to $48 \% .^{1-3}$ Non-unions are costly to treat and add a large financial burden to healthcare services worldwide. ${ }^{4}$ Various options are available to treat tibial shaft non-unions. For aseptic tibial nonunions with an intramedullary nail (IMN) in situ, an exchange nail is an excellent treatment option. ${ }^{5}$ With other failed initial treatment modalities, the use of a circular external fixator with or without bone grafting and a fibular osteotomy is a popular and successful treatment modality. ${ }^{6}$ Yet, although high rates of union can be achieved, this option is not always well tolerated by patients ${ }^{6-8}$ and is associated with high costs. Frequent outpatient visits are often necessary, which makes regular follow-up challenging, especially for patients with low incomes or long travel distances to the hospital. Lastly, some patients are reluctant to undergo external fixator treatment, especially those who previously had treatment with an external fixator.

Literature is limited on the use of IMN as treatment for non-union of the tibia in cases which have not previously been treated with a nail, $2,8,9$ as percutaneous IMN insertion across a non-union is challenging and time-consuming.

The suprapatellar approach is a recent variation of the traditional infrapatellar approach for the insertion of a tibial nail. The suprapatellar approach allows insertion of the nail in an extended knee, which aids correction of malalignment in the sagittal and coronal planes. ${ }^{10-13}$ It also creates a straight working channel, allowing the passage of rigid, straight reamers to cross the nonunion site, facilitating IMN for shaft non-unions not previously treated with IMN. Currently there is no literature available on the use of this technique to treat non-unions of the tibia.

The aim of this study was to evaluate the outcome in the form of rate and time of union and complications of a series of non-unions treated with suprapatellar entry IMN.

\section{Patients and methods}

Patients with an aseptic tibial non-union, who were treated with a suprapatellar entry IMN from May 2016 to April 2018, were reviewed retrospectively.

We included non-unions of diaphyseal tibial fractures initially treated with a cast, external fixator or plate. Patients initially treated with an IM tibial nail, non-union of peri-articular tibial fractures and non-unions with signs of active sepsis were excluded. Active infection was defined as the presence of a draining sinus or local clinical signs of infection. Inflammatory markers were not routinely used to exclude infection. Suspected active infection, using these parameters, was diagnosed by an experienced limb reconstruction surgeon. Patients younger than 18 years of age were also excluded.

Patients in this series were referred to a tertiary care limb reconstruction unit in Cape Town, South Africa. Ten (out of 13) patients had their index treatment for the tibia fracture at referral units in district or secondary care facilities.

Demographic data such as age and sex, as well as risk factors for non-union such as smoking, vitamin D deficiency and open fractures, were recorded. Open fractures were graded according to the Gustilo-Anderson classification. ${ }^{14}$ Modifiable risk factors, such as smoking and vitamin $D$ deficiency, were addressed as per our unit protocol.

\section{Definition and classification}

A non-union was defined as a fracture which has not healed within six months of treatment and is unlikely to heal without further intervention. ${ }^{15}$ The diagnosis of a non-union was based on the clinical and radiological assessment by two orthopaedic surgeons.
Table I: RUST scoring system ${ }^{16,17}$

\begin{tabular}{|l|l|l|}
\hline Score per cortex & \multicolumn{1}{|c|}{ Callus } & \multicolumn{1}{c|}{ Fracture line } \\
\hline 1 & Absent & Visible \\
\hline 2 & Present & Visible \\
\hline 3 & Present (bridging) & Invisible \\
\hline
\end{tabular}

Radiological union was graded using the radiographic union score for tibial fractures (RUST) score on post-operative radiographic films. ${ }^{16,17}$ According to Whelan et al., ${ }^{16}$ a score is assigned to each cortex on an anteroposterior and lateral X-ray, based on the assessment of healing at each cortex (Table I). The individual scores are added. A minimum of 4 indicates a definite non-union and a maximum of 12 indicates a definite union.

Functional union was defined by the ability of the patient to weight bear on the treated leg without the use of an assistive device and without experiencing pain. 'Stiff' and 'mobile' non-unions were identified on a clinical basis according to the llizarov classification.

\section{Procedure}

Treatment with a tibial nail was offered to patients with a mobile non-union without a significant bone defect or in stiff non-unions without a significant deformity. If an external fixation device was used to treat the initial fracture, it was removed prior to the index procedure. No exchange from external fixation to intramedullary fixation was performed in a single sitting. The mean time from removal of external fixator to insertion of an intramedullary nail was 3 months (range1-7).

A reamed suprapatellar entry IMN was used (Metanail, Smith \& Nephew, Memphis, Tennessee).$^{18}$ For this, the patient was positioned supine on a radiolucent table with the knee flexed at $10-20^{\circ}$. A $3-5 \mathrm{~cm}$ midline incision was used extending from the superior pole of the patella proximally. The quadriceps muscle was divided or mobilised to gain access to the patellofemoral joint, thus establishing the suprapatellar portal.

Tibia alignment was achieved with the use of blocking or Poller screws if necessary. Three patients required a fibula osteotomy at the same sitting for which a $10 \mathrm{~mm}$ section of fibula was excised using an oscillating saw. A fibular osteotomy was only performed if the fibula was united.

The non-union site was not opened and bone graft was not added in any cases. A set of solid, elastic reamers was used to cross the non-union site.

In all cases the fracture site was compressed. This controlled compression was achieved by performing distal locking first, followed by utilising the dynamic compression tool of the nail ${ }^{19}$ (Figure 1).

Medullary tissue samples were routinely collected from the intramedullary reaming and sent for microscopy, culture and sensitivity (MCS) in all cases.

\section{Post-operative management}

Patients were mobilised with partial weight bearing as tolerated from day 1 post-surgery. Physiotherapy was initiated to maintain knee and ankle range of motion.

Cases with subclinical infection confirmed with intra-operative tissue cultures were treated with at least six weeks of culturespecific antibiotics. This included an agent active against biofilmbased infections (rifampicin in Gram-positive infections if sensitive; ciprofloxacin in Gram-negative infection if sensitive). Of note is that these cases did not meet the exclusion criteria of this study, as the infection was not active at time of surgery, but subclinical.

Patients were routinely followed up with radiographs every six weeks until union (Figure 2). 

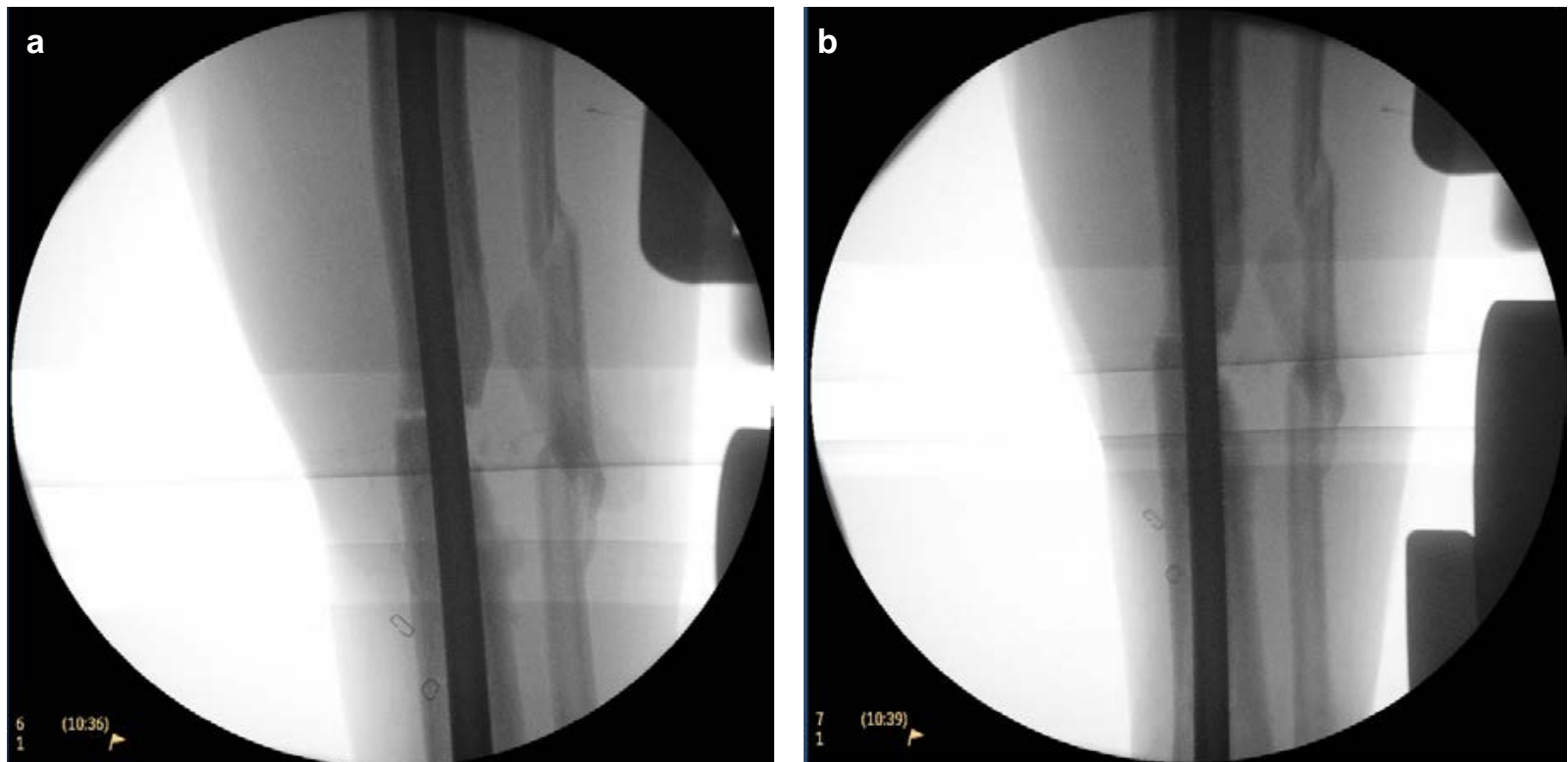

Figure 1. Controlled compression. Fluoroscopic picture a) before and, b) after compression. Note the obliteration of the fracture gap.
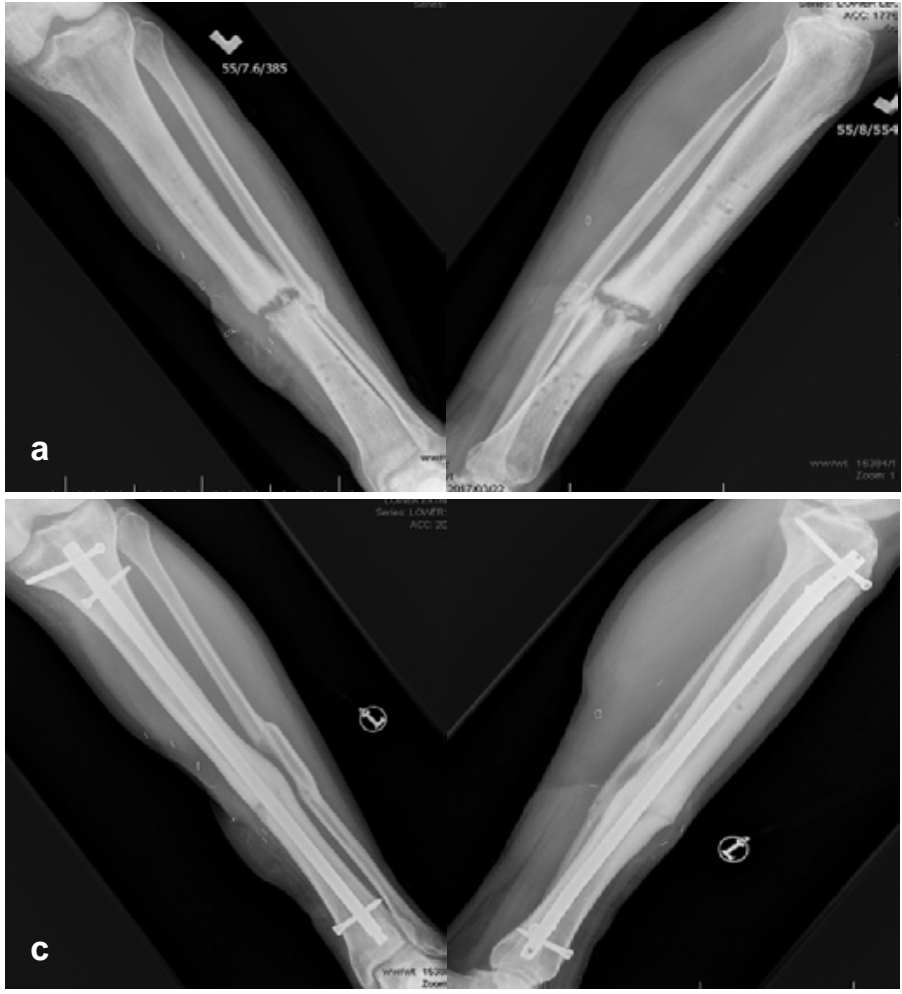

\section{Results}

Thirteen cases were included for review. Twenty-six tibial nonunions were excluded because of treatment using other treatment modalities. Characteristics and treatment of the study group are listed in Table II. All patients achieved functional and radiological union without further intervention. The mean time to radiological union was 5.8 months. All patients were followed up until union was achieved. The median length of follow-up was 7 months (interquartile range 6.5 months) (Table III).

\section{Complications/sepsis}

Three patients grew a positive bacterial culture on tissue taken at time of surgery. In two this was a methicillin-sensitive

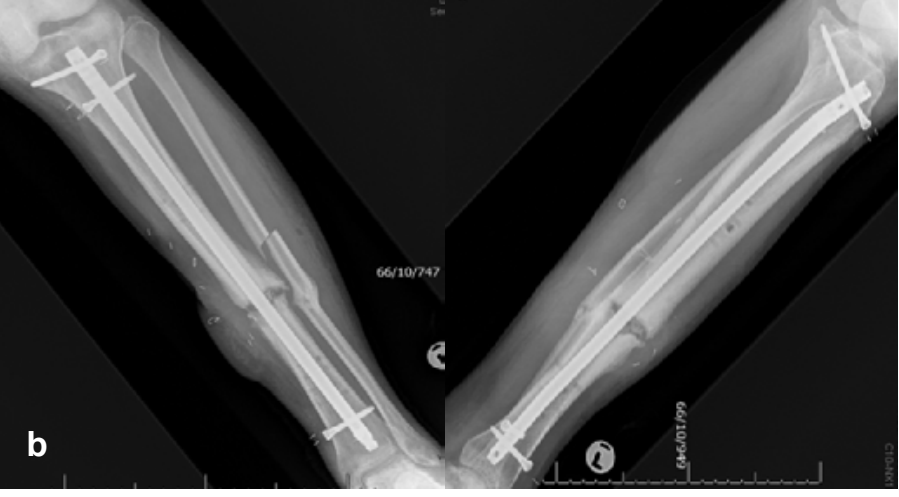

Figure 2. A 30-year-old male sustained an open tibia fracture and was initially managed with a biplanar external fixation. Other risk factors for his tibia non-union included substance abuse, smoking and a low vitamin D level. He had positive intra-operative cultures but responded well to culture-specific antibiotics. Films taken a) before suprapatellar tibial nail; b) day 1 postoperatively; and c) final films, indicating bony union

Staphylococcus aureus species (MSSA), and one culture result was positive for a Morganella species as well as an MSSA species. All three patients were treated with culture-specific antibiotics for at least six weeks. Of these three patients, two developed implant sepsis, evidenced by a draining sinus. The third patient did not develop wound complications, nor signs of implant sepsis and was therefore not considered a complication. The implant sepsis resolved in one of the two patients after completion of an antibiotic course, and his implant was removed following union. This patient had no signs of chronic osteitis at his last follow-up. The other patient developed chronic osteitis. This was treated with intermittent suppressive antibiotics until union, after which the implant was removed, followed by reaming and an antibiotic cement nail implantation. Of note is this patient previously had chronic osteitis and the non-union was the result of a pathological fracture through 
Table II: Characteristics and treatment of the study group

\begin{tabular}{|c|c|c|c|c|c|c|}
\hline No. & Age (years) & Sex & Initial treatment & Classification & Risk factors for non-union & Operation \\
\hline 1 & 40 & Male & Circular frame & Mobile & $\begin{array}{l}\text { Gr } 3 \text { open fracture } \\
\text { Vit D deficient }\end{array}$ & SP IM nail \\
\hline 2 & 32 & Male & Circular frame & Stiff & Gr 3 open & SP IM nail \\
\hline 3 & 29 & Male & Cast & Stiff & $\begin{array}{l}\text { Gr } 3 \text { open fracture } \\
\text { Smoker }\end{array}$ & SP IM nail \\
\hline 4 & 43 & Female & Circular frame & Mobile & Vit $D$ deficient & SP IM nail \\
\hline 5 & 29 & Male & Circular frame & Stiff & Vit $D$ deficient & SP IM nail \\
\hline 6 & 43 & Female & Cast & Stiff & $\begin{array}{l}\text { Smoker } \\
\text { Previous chronic osteomyelitis } \\
\text { Non-compliance }\end{array}$ & Fibular osteotomy \& SP IM nail \\
\hline 7 & 36 & Male & Circular frame & Mobile & $\begin{array}{l}\text { Gr } 3 \text { open fracture } \\
\text { Failed Masquelet technique }\end{array}$ & SP IM nail \\
\hline 8 & 30 & Male & Biplanar ex-fix & Stiff & $\begin{array}{l}\text { Gr } 3 \text { open fracture } \\
\text { Vit D deficient } \\
\text { Smoker } \\
\text { Cannabis }\end{array}$ & Fibular osteotomy \& SP IM nail \\
\hline 9 & 50 & Male & Circular frame & Mobile & $\begin{array}{l}\text { Gr } 3 \text { open fracture } \\
\text { Smoker }\end{array}$ & Fibular osteotomy \& SP IM nail \\
\hline 10 & 34 & Male & Biplanar ex-fix & Mobile & $\begin{array}{l}\text { Gr } 3 \text { open } \\
\text { Smoker } \\
\text { Cannabis }\end{array}$ & SP IM nail \\
\hline 11 & 30 & Male & Cast & Mobile & GSW injury with large zone of comminution & SP IM nail \\
\hline 12 & 29 & Male & Circular frame & Mobile & Gr 3 open fracture & SP IM nail \\
\hline 13 & 40 & Male & Circular frame & Mobile & None & SP IM nail \\
\hline
\end{tabular}

Gr: grade; SP: supra-patellar; IM: intramedullary; GSW: gunshot wound

Non-union classification according to the llizarov clinical assessment; open fracture grading done according to the Gustilo-Anderson classification ${ }^{14}$

Table III: Results and outcomes of study group

\begin{tabular}{|c|c|c|c|c|c|}
\hline No. & $\begin{array}{l}\text { Time to union } \\
\text { (months) }\end{array}$ & $\begin{array}{l}\text { Length of follow-up } \\
\text { (months) }\end{array}$ & $\begin{array}{l}\text { Complication } \\
\text { (Y-Yes/N-No) }\end{array}$ & Details & Further surgery \\
\hline 1 & 3 & 4 & $\mathrm{~N}$ & & None \\
\hline 2 & 5 & 5 & $\mathrm{~N}$ & & None \\
\hline 3 & 5 & 5 & $\mathrm{~N}$ & & None \\
\hline 4 & 8 & 8 & $\mathrm{~N}$ & & None \\
\hline 5 & 7 & 7 & $\mathrm{~N}$ & & None \\
\hline 6 & 4 & 17 & $Y$ & $\begin{array}{l}\text { Chronic osteomyelitis quiescent } \\
\text { after treatment }\end{array}$ & $\begin{array}{l}\text { Removal of infected nail at union, } \\
\text { reaming and antibiotic cement nail }\end{array}$ \\
\hline 7 & 8 & 11 & $\mathrm{~N}$ & & None \\
\hline 8 & 6 & 13 & $\mathrm{~N}$ & $\begin{array}{l}\text { Positive intra-operative cultures } \\
\text { No signs of implant sepsis }\end{array}$ & Implant removal \\
\hline 9 & 5 & 5 & $\mathrm{~N}$ & & None \\
\hline 10 & 4 & 12 & $Y$ & $\begin{array}{l}\text { Positive intra-operative cultures } \\
\text { Implant sepsis }\end{array}$ & Implant removal \\
\hline 11 & 5 & 6 & $\mathrm{~N}$ & & None \\
\hline 12 & 7 & 7 & $\mathrm{~N}$ & & None \\
\hline 13 & 3 & 3 & $\mathrm{~N}$ & & None \\
\hline
\end{tabular}

the site of chronic osteitis. Consideration was given to treat this non-union with a circular external fixator, but the patient refused the application of an external fixator. At this patient's last follow-up (18 months) the chronic osteitis was quiescent and there were no signs of recurrence of infection (Figure 3).

No patients developed implant failure, hardware irritation or any other complication in the follow-up period.

\section{Discussion}

In this series we achieved union in all cases, using a suprapatellar entry IMN.
Limited recent literature is available regarding the use of interlocking nails for the treatment of tibial non-unions not previously treated with a nail. ${ }^{2,9}$ A reamed exchange nail is an excellent treatment option for aseptic tibia non-unions with a nail in situ. ${ }^{5}$ Reamed nailing with use of larger nails creates greater stability and is believed to provide local bone graft at the fracture or non-union site that may stimulate healing. ${ }^{3,20}$ According to Tsang et al., the union rate with exchange nailing ranges from $63 \%$ (after the first non-union procedure) to $100 \%$ following subsequent nonunion procedures. ${ }^{21}$

Megas et al. demonstrated that reamed infrapatellar entry IMN resulted in union in all included patients within a period of six 

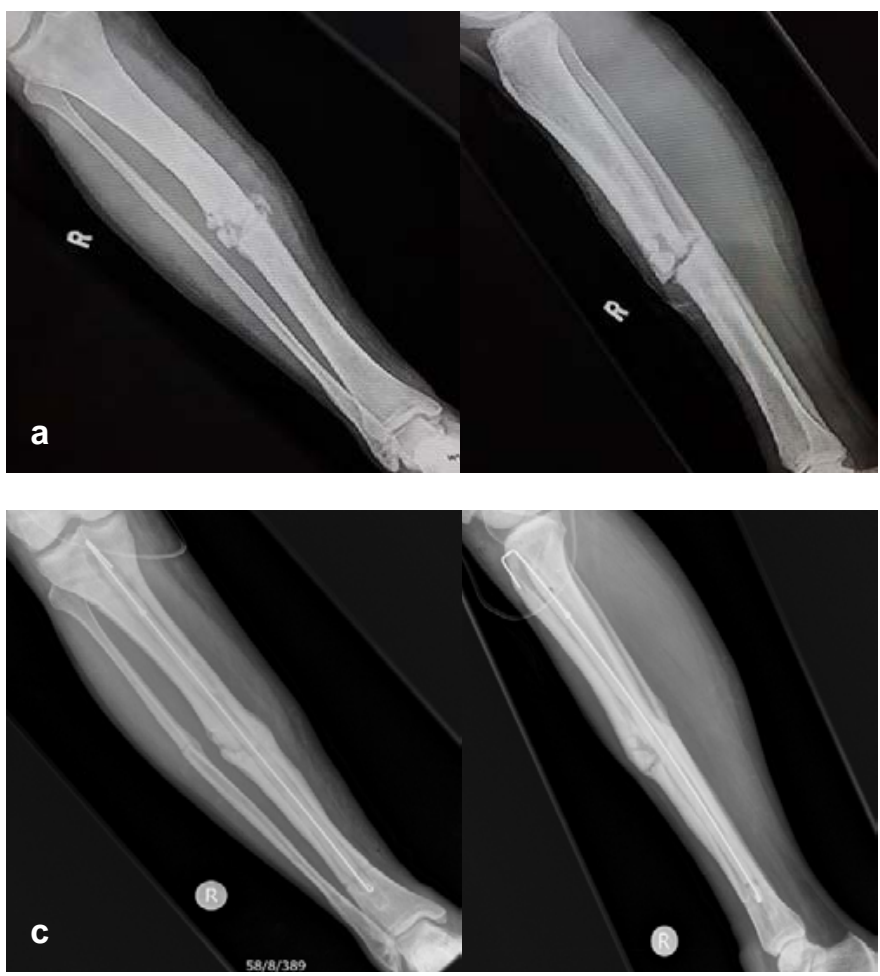
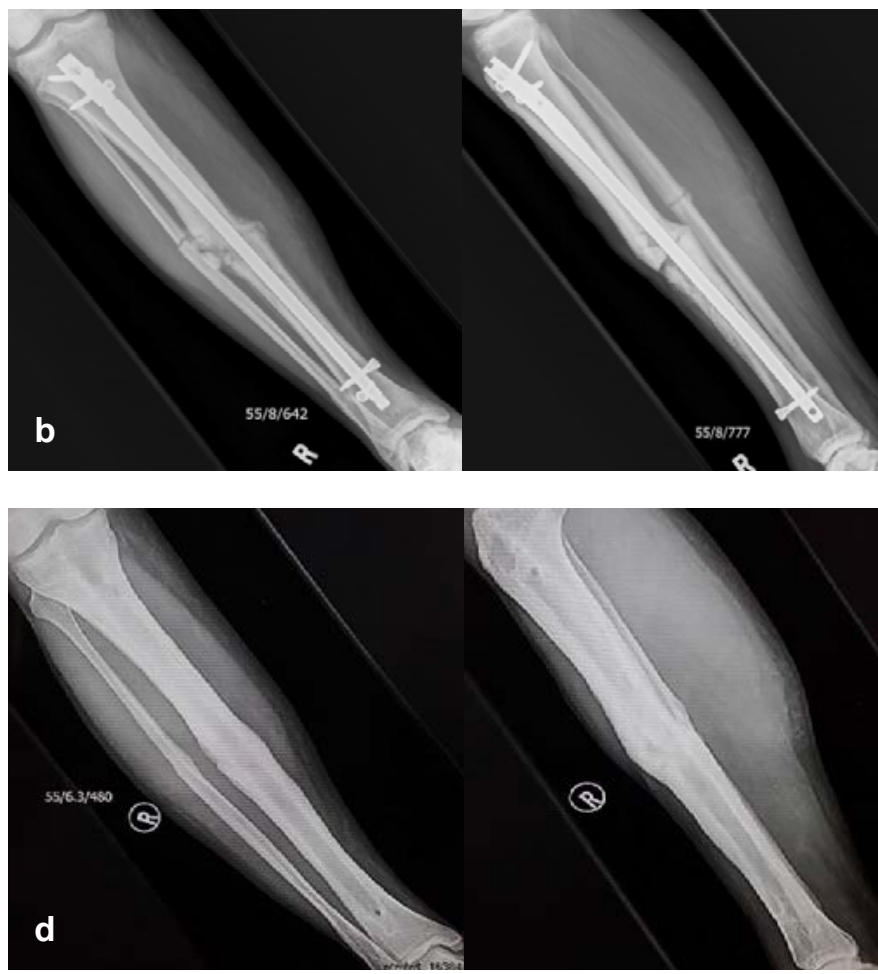

Figure 3. A 43-year-old female's tibia fracture was managed in a plaster cast. She had positive intra-operative cultures and proceeded to develop active osteitis. She required removal of her implant and the subsequent insertion of an antibiotic-impregnated cement nail and culture-specific antibiotics. The osteitis resolved without further sequelae.

Radiographic images taken a) before surgery, b) at the first follow-up consultation, c) after insertion of antibiotic impregnated cement nail and, d) at the final consultation.

months, with a low infection rate (2\%). ${ }^{2}$ Yet, in 16 of 50 cases, opening the non-union site was necessary to enable insertion of the nail.

The suprapatellar approach is a variation on the standard infrapatellar nail. The advantages of the suprapatellar nail above the standard nail include easier and improved tibial alignment, improved post-operative knee range of motion and a decrease in the incidence of anterior knee pain. No additional complications with the use of the suprapatellar approach had been proven in the literature..$^{10-13}$

Crossing the non-union site is difficult in some cases. It is necessary to have specialised equipment available in the form of solid flexible reamers. We managed closed insertion of the nail in all cases which was facilitated by the suprapatellar nail entry, enabling a straight working channel for reamers in knee extension and facilitating access for intra-operative fluoroscopy.

A potential advantage of avoiding extensive debridement of the non-union site by opening it, might be that the 'biology' remains undisturbed and, especially with local bone grafting caused by reaming the medullary canal, this might assist healing.

By adding controlled compression at the non-union site, the stability is further increased and as such, union was achieved in both hypertrophic and atrophic/oligotrophic non-unions alike by addressing the stability at the non-union site. 19,22,23

\section{Complications}

Of the three patients with positive intra-operative cultures, two (15\%) developed signs of implant sepsis which persisted in one patient. This was subsequently successfully treated with further intervention. At the final follow-up all infections were quiescent and all patients with treated non-unions had united. Non-union and chronic sepsis often co-exist in a similar environment and these complications were not specific to the suprapatellar approach.

We acknowledge the limitations of this study. This was a retrospective single centre study with a small sample size. Due to the novel nature of this treatment option and heterogeneity of cases, a large prospective study was not feasible in our setting but could be considered as a multicentre trial in future.

\section{Conclusion}

In cases of tibial shaft non-union, without signs of active sepsis, not previous managed with a nail, suprapatellar entry IMN is a safe and reliable treatment option. The use of the suprapatellar approach makes the surgery technically easier, achieving a high union rate with an acceptable low complication rate.

\section{Ethics statement}

The authors declare that this submission is in accordance with the principles laid down by the Responsible Research Publication Position Statements as developed at the 2nd World Conference on Research Integrity in Singapore, 2010.

Prior to commencement of the study ethical approval was obtained from the ethical review board: HREC reference number 315/2018.

All procedures were in accordance with the ethical standards of the responsible committee on human experimentation (institutional and national) and with the Helsinki Declaration of 1975 , as revised in 2008

\section{Declaration}

The authors declare authorship of this article and that they have followed sound scientific research practice. This research is original and does not transgress plagiarism policies.

\section{Author contributions}

NB contributed to the conception and design of the work; the acquisition, analysis and interpretation of the data for the work; drafting the work, and submitting the final version to be published. 
SG contributed to the acquisition of data for the work, revising it critically for important intellectual content and final approval of the version to be submitted to the journal. $\mathrm{MH}$ contributed to revising it critically for important intellectual content and final approval of the version to be published.

$\mathrm{ML}$ contributed to the conception and design of the work; the acquisition, analysis and interpretation of the data for the work; drafting the work, and revising it critically for important intellectual content.

\section{ORCID}

Botma N (iD http://orcid.org/0000-0002-6053-814X

Graham S id http://orcid.org/0000-0002-4091-7548

Held M (D) http://orcid.org/0000-0002-0671-0439

Laubscher M (iD) http://orcid.org/0000-0002-5989-8383

\section{References}

1. Ferreira N, Marais L, Aldous C. The pathogenesis of tibial non-union. SA Orthop J. 2016;15(1):51-59.

2. Megas $P$, Panagiotopoulos E, Skriviliotakis S, Lambiris E. Intramedullary nailing in the treatment of aseptic tibial nonunion. Injury. 2001;32(3):233-39.

3. Bhandari et al. Randomized trial of reamed and unreamed intramedullary nailing of tibial shaft fractures. J Bone Joint Surg Am. 2008;90(12):2567.

4. Hak DJ, Fitzpatrick D, Bishop JA, Marsh JL, Tilp S, Schnettler R, et al. Delayed union and nonunions: epidemiology, clinical issues, and financial aspects. Injury. 2014;45(Suppl 2):S3-7.

5. Brinker MR, O'Connor DP. Exchange nailing of ununited fractures. J Bone Joint Surg Am. 2007;89(1):177-88.

6. Ferreira N, Marais LC. Management of tibial non-unions according to a novel treatment algorithm. Injury. 2015;46(12):2422-27.

7. Wiss DA, Stetson WB. Tibial nonunion: treatment alternatives. $J$ Am Acad Orthop Surg. 1996;4(5):249-57.

8. Richmond J, Colleran K, Borens O, Kloen P, Helfet DL. Nonunions of the distal tibia treated by reamed intramedullary nailing. $J$ Orthop Trauma. 2004;18(9):603-10.

9. Rosson JW, Simonis RB. Locked nailing for nonunion of the tibia. $J$ Bone Joint Surg Br. 1992;74(3):358-61.

10. Chen X, Xu HT, Zhang HJ, Chen J. Suprapatellar versus infrapatellar intramedullary nailing for treatment of tibial shaft fractures in adults. Medicine (Baltimore). 2018;97(32):e11799.

11. Chan DS, Serrano-Riera R, Griffing $R$, Steverson $B$, Infante A, Watson $\mathrm{D}$, et al. Suprapatellar versus infrapatellar tibial nail insertion: a prospective randomized control pilot study. J Orthop Trauma. 2016;30(3):130-34.

12. Gelbke MK, Coombs D, Powell S, DiPasquale TG. Suprapatellar versus infra-patellar intramedullary nail insertion of the tibia: a cadaveric model for comparison of patellofemoral contact pressures and forces. J Orthop Trauma. 2010;24(11):665-71.

13. Sanders RW, DiPasquale TG, Jordan CJ, Arrington JA, Sagi HC. Semiextended intramedullary nailing of the tibia using a suprapatellar approach: radiographic results and clinical outcomes at a minimum of 12 months follow-up. J Orthop Trauma. 2014;28(5):245-55.

14. Kim PH, Leopold SS. Gustilo-Anderson classification. Springer; 2012.

15. Ferreira N, Marais L, Aldous C. Challenges and controversies in defining and classifying tibial non-unions. SA Orthop J. 2014;13(2):52-56.

16. Whelan DB, Bhandari $M$, Stephen $D$, Kreder $H$, McKee MD Zdero $\mathrm{R}$, et al. Development of the radiographic union score for tibial fractures for the assessment of tibial fracture healing after intramedullary fixation. J Trauma. 2010;68(3):629-32.

17. Leow J, Clement N, Tawonsawatruk T, Simpson C, Simpson A. The radiographic union scale in tibial (RUST) fractures: Reliability of the outcome measure at an independent centre. Bone and Joint Research. 2016;5(4):116-21.

18. Jakma $T$, Reynders-Frederix $P$, Rajmohan $R$. Insertion of intramedullary nails from the suprapatellar pouch for proximal tibial shaft fractures. A technical note. Acta Orthop Belg. 2011;77(6):834-37.

19. Högel F, Gerber C, Bühren V, Augat P. Reamed intramedullary nailing of diaphyseal tibial fractures: comparison of compression and non-compression nailing. Eur J Trauma Emerg $S$. 2013;39(1):73-77.
20. Hak DJ, Lee SS, Goulet JA. Success of exchange reamed intramedullary nailing for femoral shaft nonunion or delayed union. J Orthop Trauma. 2000;14(3):178-82.

21. Tsang S, Mills L, Frantzias J, Baren J, Keating J, Simpson A. Exchange nailing for nonunion of diaphyseal fractures of the tibia: our results and an analysis of the risk factors for failure. Bone Joint J. 2016;98(4):534-41.

22. Ferreira N, Marais LC, Aldous C. Mechanobiology in the management of mobile atrophic and oligotrophic tibial nonunions. J Orthop. 2015;12(Suppl 2):S182-7.

23. Elliott DS, Newman KJ, Forward DP, Hahn DM, Ollivere B, Kojima $\mathrm{K}$, et al. A unified theory of bone healing and nonunion: $\mathrm{BHN}$ theory. Bone Joint J. 2016;98-B(7):884-91. 